\title{
Crecer aprendiendo a amar(se): educación afectivo sexual
}

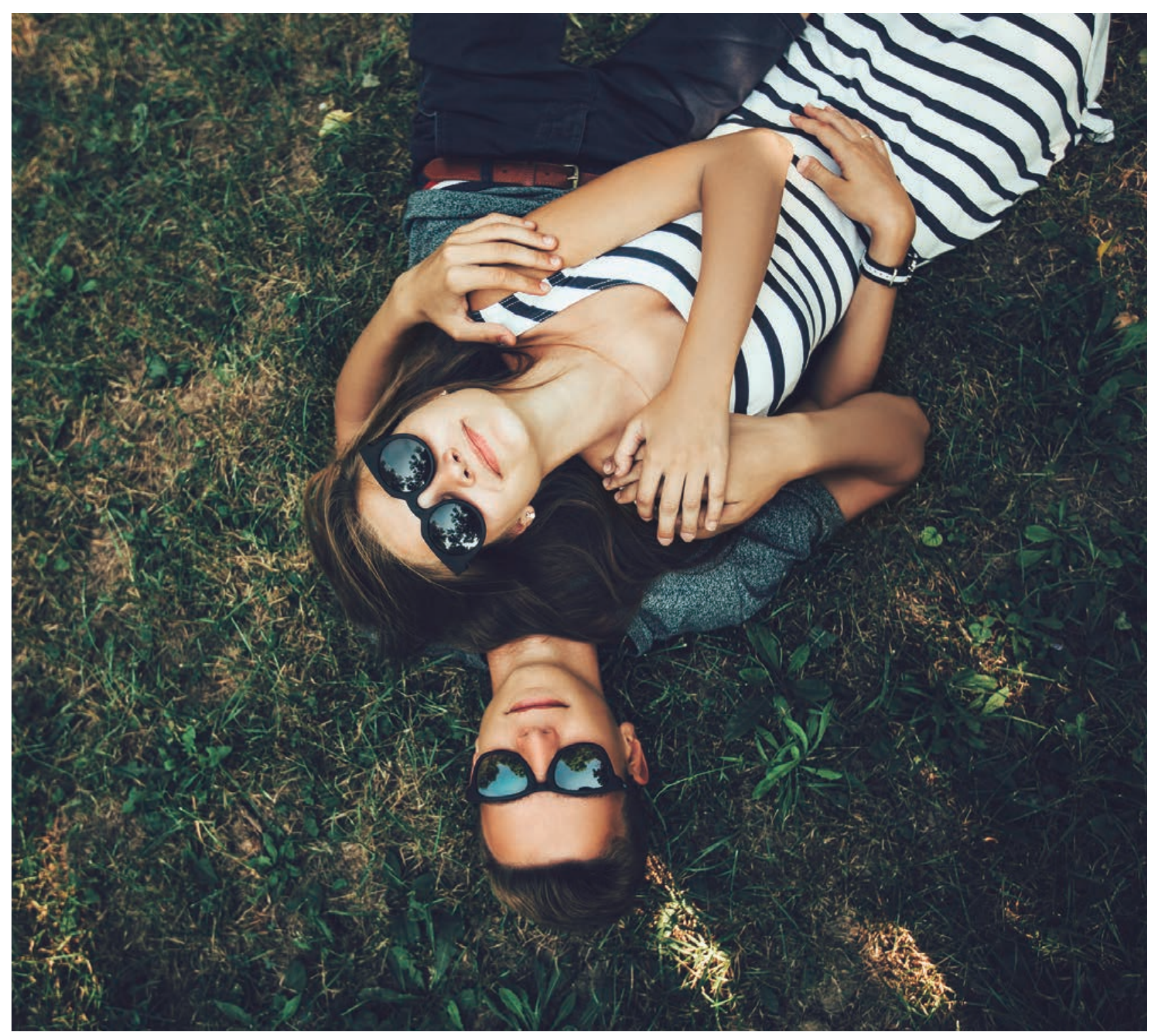

El aprendizaje afectivo-sexual más importante es tener la seguridad de que podemos querer y ser queridos, que podemos confiar en los demás y que podemos construir relaciones de

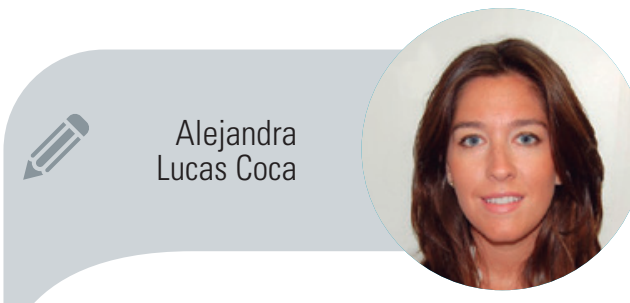

UNINPSI. Universidad Pontificia Comillas alucas@comillas.edu intimidad seguras. 
La sexualidad es una dimensión esencial en la vida de las personas que ofrece la posibilidad de disfrutar de la ternura, el placer, el afecto, la comunicación, la procreación, el compromiso, el amor, etc. Por ello es tan importante que logremos integrarla en nuestro desarrollo personal y podamos abordarla de forma abierta y positiva en el ámbito de las familias y de la escuela.

Hoy más que nunca los niños y adolescentes se ven rodeados de mensajes con un alto contenido sexual que frecuentemente llevan asociados, de forma implícita o explícita, modelos de relación negativos: machistas, violentos, carentes de ética y valores morales, etc. Felix López nos alerta de que invadiendo su infancia, de alguna manera se les está privando de ella. Al mismo tiempo se pueden sentir abrumados y sin espacios en los que poder ordenar esta información o plantear sus dudas y contradicciones acerca de la experiencia que están viviendo.

En cada generación, las familias se encuentran con el mismo reto: ofrecer a sus hijos información sobre sexualidad, con el consiguiente dilema: ¿cómo lo hago?, ¿cuánta información debo dar?, ¿será demasiado pronto?, ¿cuánto saben ya nuestros hijos?, etc.

Los padres generalmente no tienen un modelo por el que guiarse; cuando se les pregunta sobre su propia educación afectivo-sexual la mayoría dicen no haber recibido ninguna, sus padres jamás hablaron con ellos de estos temas o, si lo hicieron, no tienen claro que les gustaría tener ese mismo tipo de conversación con sus hijos. Querrían transmitir un mensaje diferente pero no saben cómo hacerlo. Con frecuencia, esto se debe a que muchos padres no tienen claro su propio posicionamiento frente a este asunto. A pesar de ello, algunos hacen la siguiente reflexión: "A mí nadie me habló de este tema o me hablaron muy poco, y tampoco me ha ido tan mal", perpetuando así el tabú.

El principal temor que se activa en los adultos y que dificulta la comunicación consiste en la posibilidad de que podrían estar adelantando inquietudes en torno a

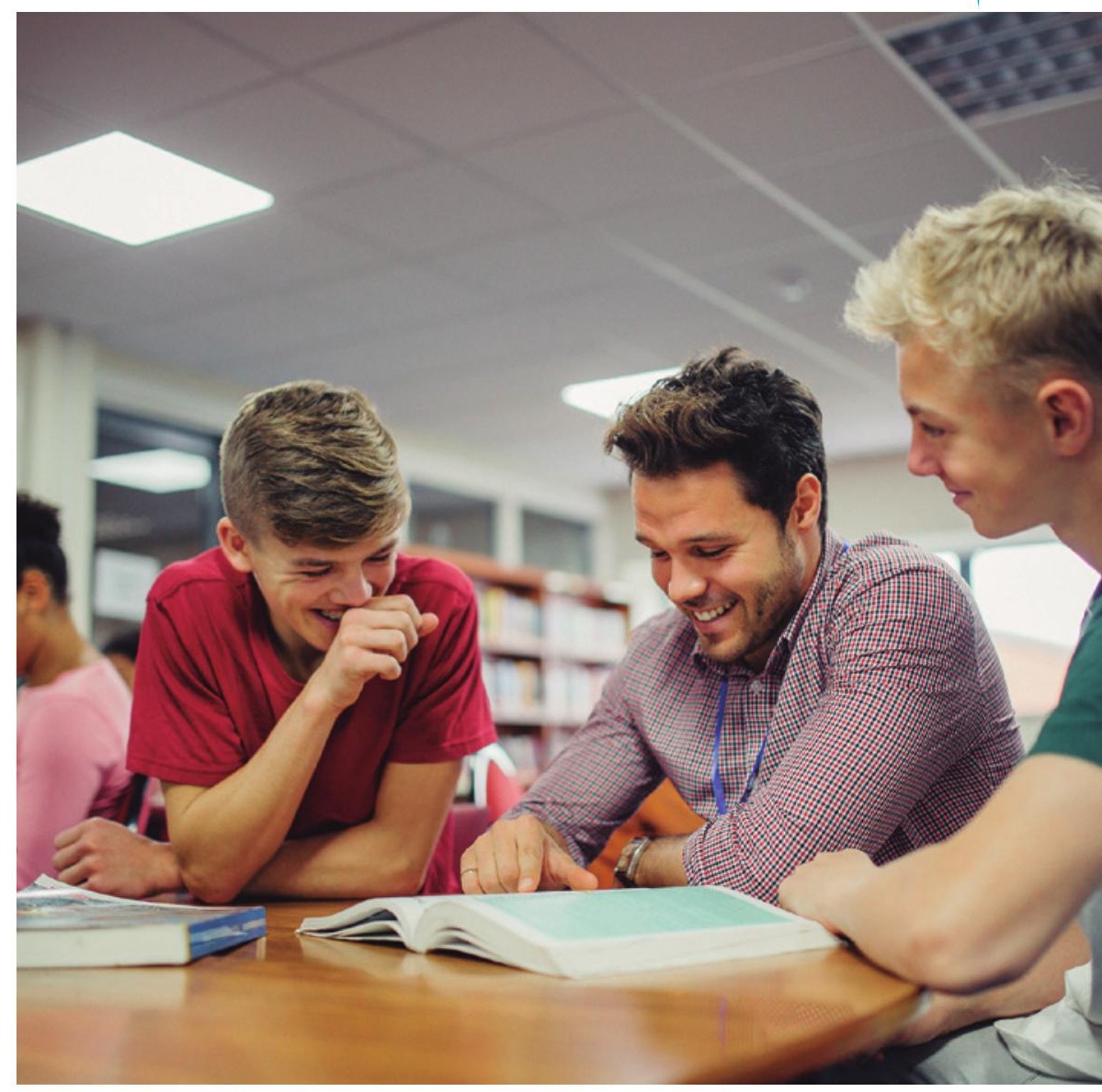

la sexualidad que no surgirían si el adulto no abordara el tema, o incluso que podrían estar estimulando su curiosidad y por tanto incitándoles a experimentar o dándoles permiso para ello.

No obstante, sabemos que cuando los menores perciben apertura para poder hablar, así como formación de calidad (y no solamente información), se sienten más seguros para poder tomar decisiones respecto a sus relaciones afectivosexuales en el presente o en el futuro. Por tanto, no es necesario esperar a que ellos pregunten o a que alcancen una determinada edad, sino que proponerles temas, estar receptivos a sus preguntas, ofrecerles información etc., les permite madurar, poner nombre a sus sentimientos y a la experiencia que rodea a la pubertad, así como dar forma a sus ideas mientras pueden explorar sus temores en un contexto seguro. Temores que, si están presentes, seguramente tratarán de resolver en otro contexto, no siempre adecuado a sus necesidades reales. Generar un espacio de comunicación y adelantarse a sus preguntas sienta un precedente: "puedes preguntar lo que 


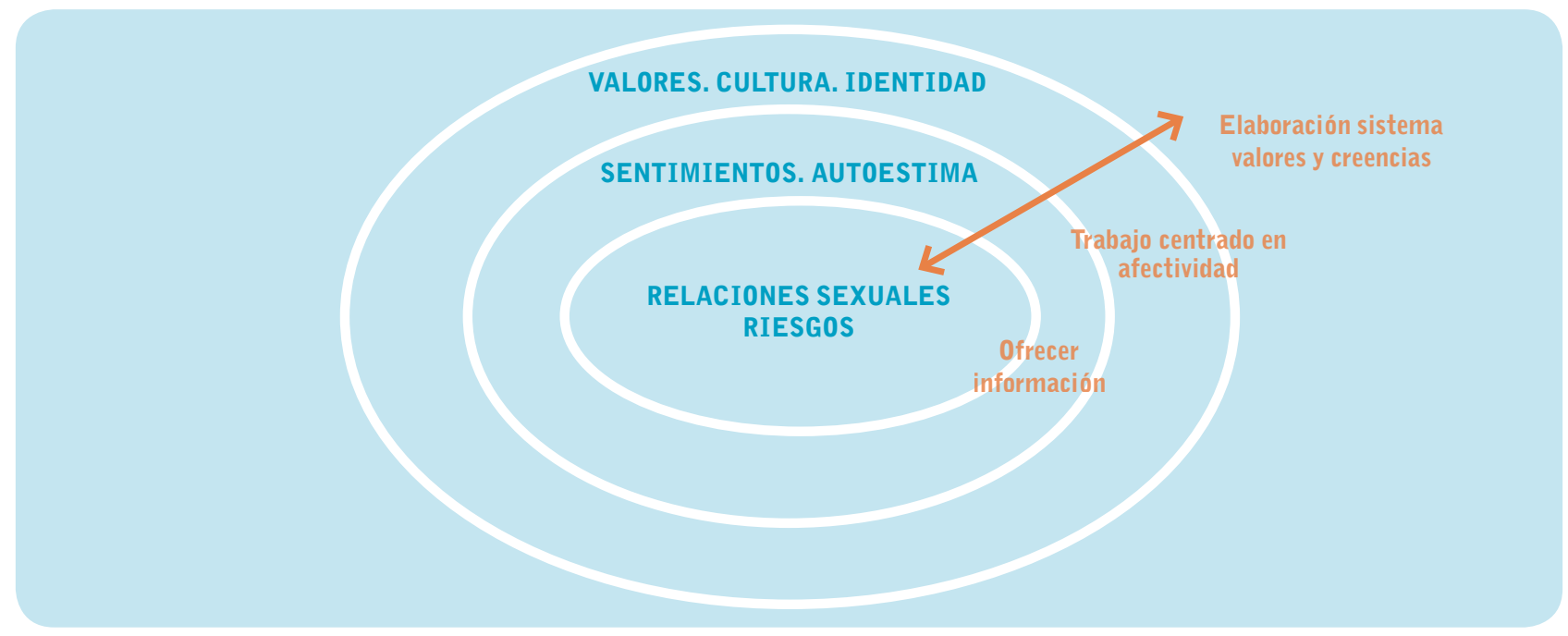

necesites". Mientras que si esperamos a que pregunten cuando lo necesiten, quizás cuando este momento llegue no sientan que existe ese espacio de confianza y seguridad para poder hablar.

Algo que inquieta y preocupa generalmente a los colegios y departamentos de orientación a la hora de organizar formaciones y programas de educación afectivo-sexual es ¿qué pensarán los padres?, ¿estarán de acuerdo con nuestra iniciativa?, o ¿cómo elegir una formación que se ajuste a los valores e ideología de las familias y de la escuela sin perder de vista la información que deben recibir los menores? Encontrar ese balance no siempre es sencillo. Pero la realidad es que a la hora de educar en sexualidad no se debería renunciar a educar en afectividad, ya que ambas son aspectos relacionados de un mismo proceso. Deberíamos entender que no se trata de enseñar a los menores a estar equipados para tener relaciones sexuales, sino que una adecuada formación es aquella que ayuda al niño y al adolescente a construirse desde el interior hacia el exterior, para poder establecer relaciones satisfactorias y positivas, que empiezan por valorarse a uno mismo y continúan tejiendo vínculos y relaciones con los otros, relaciones que en un principio serán de amistad y más adelante serán de pareja.

\section{Cuestiones de fundamento}

La educación afectivo-sexual se puede ver acompañada de programas específicos. Los programas de afectividad y sexualidad deberían responder no sólo a un derecho de los menores a ser informados sobre este tema tan importante y central en su desarrollo, sino también como un compromiso con la infancia y desde el deseo de ofrecer a las familias la oportunidad de generar espacios de diálogo diferentes.

José Antonio Marina nos recuerda que "todos somos responsables de la educación de los niños que se crían en nuestro grupo social" y "todos, cada uno desde su puesto en la sociedad, debemos retomar nuestro papel y enseñar a vivir". Esta última parte es esencial en el marco de la educación afectivo-sexual, ya que como adultos nuestro papel no es sólo ofrecer información acerca de las relaciones sexuales y los riesgos, pues sería muy reduccionista. Un desarrollo armónico de la afectividad y la sexualidad significa aprender a vivir, a relacionarse, conocerse a sí mismo, conocer a los demás, etc.

A continuación se resumen algunas de las cuestiones de fundamento que deberían guiar este tipo de formaciones:

7 Proteger y enseñar son funciones de la familia y de la escuela. Al comienzo de la vida los niños necesitan que los adultos les ayudemos a conocer e interpretar el mundo, que se lo "traduzcamos". Necesitan ser cuidados, pero también necesitan que les enseñemos a cuidarse: esto cobra especial importancia en las relaciones afectivas. Esta situación de dependencia del adulto, convoca no sólo a sus familiares y profesores sino que contemplamos, por tanto, estos programas desde una filosofía de servicio al considerar que todos los adultos somos 
miembros del sistema de cuidado y protección de los niños.

خ Ser aliados de la familia y del colegio. El equipo del programa de afectividad y sexualidad se debería integrar como un elemento más del universo del niño o alumno y pasar a formar parte, temporalmente, del sistema en el que el niño crece. La idea es alejarse de modelos formativos que se presentan como agentes externos, y tratar de construir modelos cooperativos que, además de ofrecer información objetiva, ayuden a construir puentes entre la familia, la escuela y los hijos o alumnos. No somos sustitutos de la familia o de la escuela, sino que trabajamos unidos en una misma dirección.

入 Escuela, familia y programa de educación afectivo-sexual deben ser congruentes en su mensaje, para no provocar disonancias cognitivas ni emocionales en los niños y adolescentes.

7 Generar espacios de intimidad, propiciar oportunidades de encuentro y crecimiento compartido en las familias y en la escuela. Partimos de una mirada amable hacia las familias. Esto significa que asumimos que habitualmente existe una base de intimidad y comunicación. La proximidad de la pubertad, el miedo a la sexualidad y a lo desconocido, pueden bloquear estas vías de comunicación. Uno de los objetivos centrales del programa debe ser la reactivación y la protección de estos canales de conexión.

入 Enseñar a los menores a poder usar de manera responsable y placentera su libertad, de tal forma que puedan construir relaciones que les hagan sentirse bien a ellos mismos y a los demás. Cada uno vive su sexualidad de manera diferente, ya que aquello que nos distingue del mundo animal es que tenemos libertad de decidir, pues nuestra sexualidad no pertenece al reino de la necesidad. Esto es especialmente interesante de trabajar con los adolescentes a la hora de abordar creencias como la supuesta

\section{Cooperación entre los sistemas que rodean al niño}

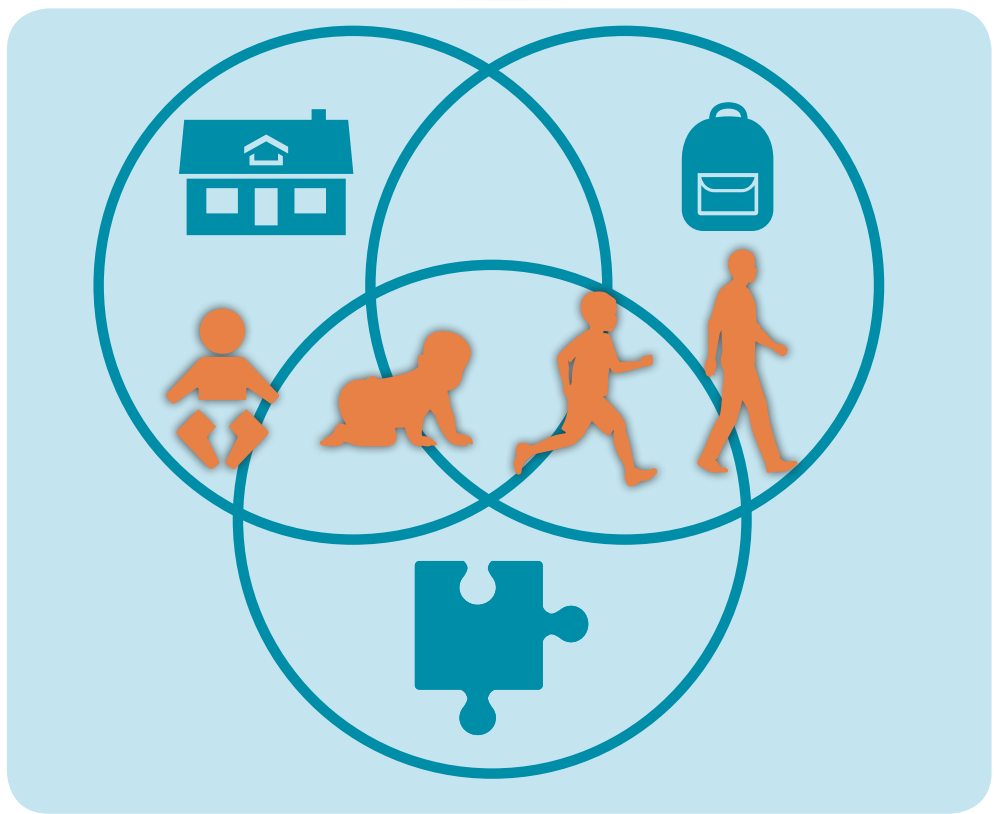

"incapacidad de controlar el deseo sexual".

- Acompañar en la elaboración de un sistema de valores y creencias, así como de un modelo amoroso sano. Precisamente porque somos libres y responsables, la sexualidad humana tiene una dimensión ética (LóPEZ, 2015). Una persona alcanza el estadio de identidad realizada tras haber atravesado momentos de cuestionamiento personal y haber sido capaz de tomar decisiones, esto debería tener lugar al final de la adolescencia. No obstante, no sucede per se con el paso del tiempo, sino que debemos ofrecer las condiciones necesarias para que se puedan desplegar los recursos para que esto suceda. Para poder construir un sistema de creencias y valores propio, y que éste sea sólido, es importante haber cuestionado los mensajes recibidos, para sentir que la decisión que uno toma es genuina.

$\boldsymbol{\lambda}$ Tener en cuenta las resonancias emocionales del adulto. Es una oportunidad poder acompañar a los jóvenes en este proceso y una gran responsabilidad. Resulta imprescindible que podamos estar en contacto con nuestra propia experiencia de la sexualidad y afectividad, nuestros valores y los sentimientos que se nos despiertan. Debemos mantener una escucha acti- 


\section{ACTIVIDADES DE AULA}

Para ayudar a los alumnos a reflexionar sobre estos temas, tan delicados e importantes, podemos pedirles al final de una sesión en el aula (de tutoría o de taller afectivosexual) que escriban:

У Algo que me gustaría preguntar sobre sexualidad y afectividad y no me atrevo a preguntar.

У 0 una pregunta sobre los cambios que estoy experimentando y otra sobre los cambios que creo que están experimentando mis compañeros del sexo contrario.

va, así como darnos cuenta de cómo impactan en nosotros las preguntas e inquietudes de los jóvenes. Hacer un buen análisis de nuestro estado nos permitirá ofrecer un espacio de seguridad y transmitirá, a la persona a la que atendemos, la sensación de que estamos ahí con ella y para ella mientras explora y organiza sus ideas. Por otro lado, nos ayudará elaborar adecuadamente nuestras respuestas.

\section{Componentes de un programa afectivo-sexual}

A la hora de implementar un programa afectivo-sexual conviene tener en cuenta ciertos componentes fundamentales:

入 Reunión con el colegio y con los tutores de cada grupo de alumnos. Esta reunión debería tener una doble función, por un lado atender la demanda del colegio y por otro, tratar de ajustar el programa a las necesidades de cada grupo de alumnos. Aunque tengamos una planificación de contenidos para cada curso escolar, la idea es poder hacer un "programa a medida". Sabemos que cada generación es diferente y que dentro de cada curso puede haber diferencias de madurez. A pesar de que no es posible adaptar el taller a cada alumno, es recomendable tratar de adecuarse a la capacidad de procesamiento y al punto de desarrollo en que se encuentre cada clase.

入 Valorar a nivel de aula el posicionamiento de los alumnos en lo que se refiere a creencias y actitudes acerca de la sexualidad. Esto nos permite ajustar los contenidos de las dinámicas y conocer qué aspectos son importantes trabajar con cada grupo, como por ejemplo los mitos del amor romántico o la presencia de los celos como signo incuestionable de amor.

त Encuentro con las familias. De la organización del colegio dependerá el formato a través del cual se haga partícipes a los padres -taller, escuela de familias o escuela de padres- pero en cualquier caso éste es un componente imprescindible. A diferencia de otros enfoques, la meta no es ofrecer únicamente información o pautas de comunicación a los padres, sino que éste es el primer paso de reactivación de las vías de comunicación. Es frecuente que las familias tengan el radar puesto en los peligros y los riesgos. Lo primero que debemos hacer es dirigirlo a sus miedos, para tratar de desbloquearlos y acercarles a sus hijos. Estos últimos manifiestan el deseo de hablar con sus padres y no saber cómo, ya que suelen recibir respuestas deslegitimadoras: consejos o prohibiciones, se sienten ridiculizados, regañados, etc. Probablemente porque hay poco tiempo para hablarlo, porque los padres creen que será su única oportunidad para decirlo y quieren que el mensaje importante quede claro (generalmente, un mensaje relativo al peligro), o porque hay muchos alumnos en clase, cuando esta respuesta viene de un profesor. Escuchar esto genera incredulidad, podemos apoyarnos en testimonios anónimos de los propios alumnos que reflejen el deseo de hablar con sus familias, si los tenemos.

入 Taller en el aula con los alumnos. El trabajo en el aula debe ser dinámico, fomentando la participación activa de los alumnos, lo que favorece el procesamiento interno. La presencia del tutor es necesaria por varios motivos: conoce a los menores y puede ayudar a identificar reacciones significativas o necesidades especiales, da coherencia a la idea de colaboración familiaescuela-programa, y permite evaluar la calidad del programa. En los cursos inferiores sería recomendable llevar a 
cabo, aproximadamente un mes después, una sesión de seguimiento, para poder abordar aquellos temas que se hayan ido elaborando en ese tiempo. Los alumnos de cursos superiores deberían tener un espacio de atención individual en el que plantear sus inquietudes.

त Escucha Individual. Este espacio de escucha es conveniente que tenga lugar en una fecha próxima a la última sesión en el aula, en el mismo día o unos días después.

\section{Los tiempos}

En todo proceso de desarrollo los "tiempos" son un factor clave. Un programa de educación afectivo-sexual no debería dar comienzo antes del segundo trimestre lectivo, por varios motivos:

$\boldsymbol{\pi}$ Los alumnos del grupo necesitan conocerse y estar relativamente cómodos para comenzar a abrirse y hablar de temas que atañen a lo más profundo de su persona. En muchos colegios se hacen cambios de grupo cada cierto tiempo, por lo que los alumnos necesitan tiempo para ubicarse y organizarse de nuevo si se diera el caso.

$\boldsymbol{\lambda}$ Es importante que el equipo de tutores y orientación pueda conocer a sus alumnos, para ayudar en la elaboración del programa y participar en las dinámicas si fuera necesario.

入 En función del curso, la programación académica nos permite que ya se hayan trabajado en biología, entre otros, temas relacionados con los cambios fisiológicos asociados a la pubertad o el sistema reproductor. Esto va activando en los alumnos la curiosidad y el cuestionamiento interno.

7 El programa llega cuando la mayoría están "preparados" para poder hablar - empezar a plantearse cuestiones acerca de la afectividad y la sexualidad. Mientras tanto, han tenido tiempo de preguntar en casa, en el colegio o entre sus iguales, ya han recorrido una parte del camino exploratorio.

\section{¿Y yo dónde estoy con este tema?}

Vuestros hijos en muchas ocasiones no saben lo que les pasa, no saben cómo preguntarlo o necesitan que alguien les ayude a traducir lo que les está sucediendo; por ejemplo cuando eran pequeños y se enfadaban podíais ayudarles, les explicabais que lo que sentían era enfado y qué podían hacer con él. Ahora podría estar sucediéndoles lo mismo, pero al aparecer la palabra 0 el contexto SEXUALIDAD parece que todo se complica, y sin embargo necesitan vuestra ayuda más que nunca aunque de manera diferente. Antes de lanzarnos a responder es importante que podamos darnos cuenta de cómo resonamos nosotros a nivel emocional con cada uno de estos temas, qué inquietudes nos genera hablar de ciertos temas, si nos resulta fácil o difícil y tratar de explorar por qué. "Cómo descubrí yo esto a la edad de mi hijo, dónde obtuve la información, qué me habría gustado saber, qué fue lo que me resultó más incómodo preguntar..." Poder reconocer nuestro estado emocional nos permitirá conectar con el de nuestros hijos.

\section{Componentes de un programa de educación afectivo-sexual}

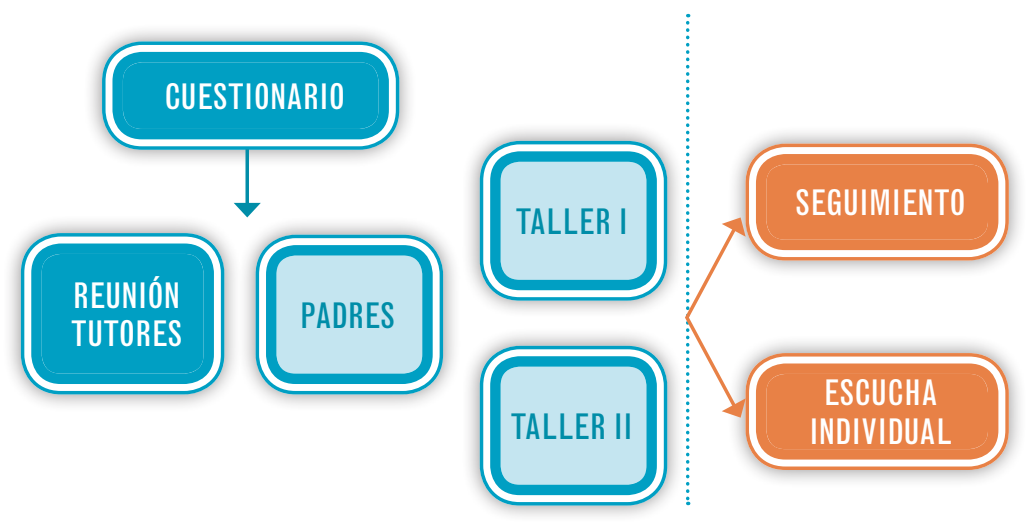

Para poder ofrecer un programa educativo de esta naturaleza, es necesario un conocimiento e interés profundo por el ser humano y su desarrollo psicoafectivo. Puede ser impartido por el propio centro escolar cuando cuenta con profesionales adecuadamente preparados. Otras veces, se puede recurrir a programas desarrollados por centros especializados en la orientación familiar, en la terapia familiar, así como en la intervención con niños y adolescentes; desde la Unidad de Intervención Psicosocial (UNINPSI) de la Universidad Pontificia Comillas venimos desarrollando e impartiendo un programa de afectividad y sexualidad en los colegios: Crecer Aprendiendo a Amar(se), que se desarrolla basado en estos principios y componentes.

En un momento $u$ otro de nuestras vidas todos deseamos amar y ser amados, de pequeños buscamos el amor de nues- 


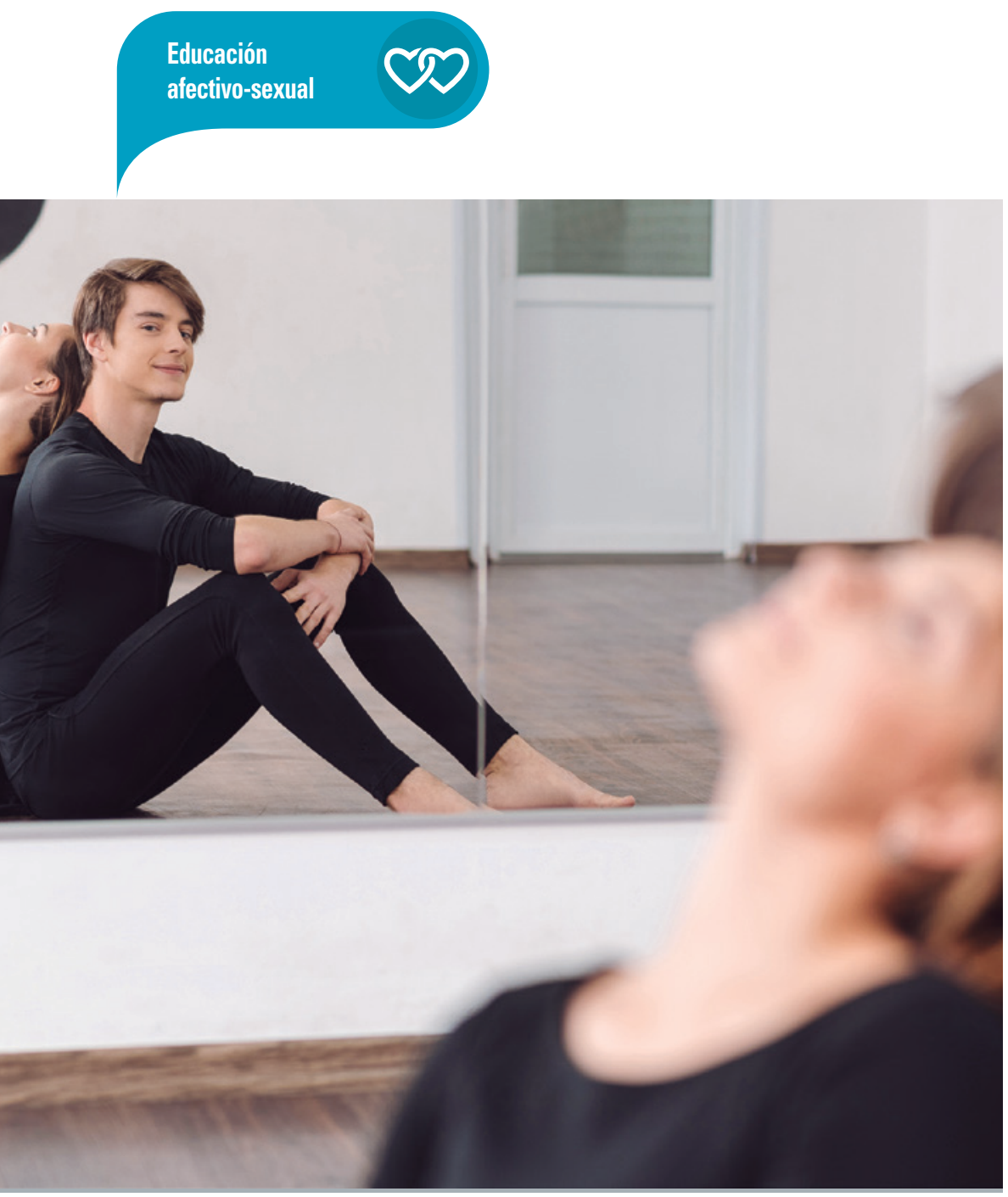

Una adecuada formación es aquella que ayuda al niño y al adolescente a construirse desde el interior hacia el exterior, para poder establecer relaciones salisfactorias y positivas, que empiezan por valorarse a uno mismo y continúan tejiendo vínculos con los otros

tros padres, luego queremos ser aceptados por nuestros amigos, y más adelante empezamos a sentir que el destino de ese amor es una persona concreta. Pero la gran pregunta que todos nos hemos hecho alguna vez es: ¿cómo sé si lo que siento es amor? Aquí es donde intervienen respuestas del tipo: "no te preocupes, cuando llegue lo sabrás", "cuando aparezca lo tendrás claro", "en ese momento no dudarás". Respuestas bien intencionadas, pero que lamentablemente no calman ni reducen la angustia o la incertidumbre.

Con la llegada de la pubertad, algunas partes del cuerpo comienzan a cambiar y adquieren un significado distinto. Manifiestan la sexualidad. Sin embargo, esto no tiene nada que ver con los sentimientos, el interés sexual o la madurez emocional. Teóricamente, cuando termina el desarrollo de la pubertad nuestro cuerpo está preparado biológicamente para la reproducción, pero ¿esto quiere decir que estamos nosotros preparados? Esta es una idea que podemos y debemos pensar con los adolescentes.

Estar ahí, con ellos, cuando se plantean estas preguntas es un privilegio. A pesar de las apariencias, no desean recorrer ese camino completamente solos. Necesitan que alguien les guíe y les ayude, a su ritmo, pero cerca. La influencia de la familia y de la escuela en la formación de las actitudes hacia la sexualidad y las relaciones afectivas determinará que los jóvenes tengan una visión positiva y valiosa de las mismas o, por el contrario, que las vivan como algo peligroso o dañino. Teniendo en cuenta que el aprendizaje afectivo-sexual más importante consiste en descubrir que podemos amar y ser amados, cabría hacerse la siguiente reflexión ¿qué lugar quiero que ocupe esto en la vida de mis hijos y alumnos? •

\section{(D.) DARA SABER MÂS}

Gómez-Zapiain, J. (2009). Apego y Sexualidad. Entre el vínculo afectivo y el deseo sexual. Madrid: Alianza Editorial.

LóPEZ, F. (2005). La educación sexual de los hijos. Madrid: Pirámide Somers, L., y Somers, B. (1990). Cómo hablar a sus hijos sobre el amor y el sexo. Barcelona: Paidós.

\section{HEMOS HABLADO DE}

\section{Educación; afectividad; sexualidad; familia; escuela.}

Este artículo fue solicitado por PADRES Y MAESTROS en mayo de 2017, revisado y aceptado en octubre de 2017. 